\title{
Privacy-Preserved Pseudonym Scheme for Fog Computing Supported Internet of Vehicles
}

\author{
Jiawen Kang ${ }^{\circledR}$, Rong Yu, Member, IEEE, Xumin Huang, and Yan Zhang ${ }^{\circledR}$, Senior Member, IEEE
}

\begin{abstract}
As a promising branch of Internet of Things, Internet of Vehicles (IoV) is envisioned to serve as an essential data sensing and processing platform for intelligent transportation systems. In this paper, we aim to address location privacy issues in IoV. In traditional pseudonym systems, the pseudonym management is carried out by a centralized way resulting in big latency and high cost. Therefore, we present a new paradigm named Fog computing supported IoV (F-IoV) to exploit resources at the network edge for effective pseudonym management. By utilizing abundant edge resources, a privacy-preserved pseudonym $\left(P^{3}\right)$ scheme is proposed in F-IoV. The pseudonym management in this scheme is shifted to specialized fogs at the network edge named pseudonym fogs, which are composed of roadside infrastructures and deployed in close proximity of vehicles. $P^{3}$ scheme has following advantages: 1) context-aware pseudonym changing; 2) timely pseudonym distribution; and 3) reduced pseudonym management overhead. Moreover, a hierarchical architecture for $\boldsymbol{P}^{\mathbf{3}}$ scheme is introduced in F-IoV. Enabled by the architecture, a context-aware pseudonym changing game and secure pseudonym management communication protocols are proposed. The security analysis shows that $P^{3}$ scheme provides secure communication and privacy preservation for vehicles. Numerical results indicate that $P^{3}$ scheme effectively enhances location privacy and reduces communication overhead for the vehicles.
\end{abstract}

Index Terms - Internet of vehicles, fog computing, location privacy, pseudonym management, game theory.

\section{INTRODUCTION}

$\mathbf{W}$ ITH rapid development of wireless communication technologies, Internet of Vehicles (IoV) has been an indispensable platform with information interaction among vehicles, humans, and road-side infrastructures. IoV improves road safety and traffic efficiency, which significantly facilitates the implementation of Intelligent Transport System (ITS) [1].

Manuscript received December 17, 2016; revised May 29, 2017 and August 27, 2017; accepted October 3, 2017. This work was supported in part by programs of NSFC under Grant 61422201, Grant 61370159, Grant U1301255, and Grant U1501251, in part by the Science and Technology Program of Guangdong Province under Grant 2015B010129001, in part by the Special-Support Project of Guangdong Province under Grant 2014TQ01X100, in part by the Science and Technology Program of Guangzhou under Grant 2014J2200097, and in part by projects 240079/F20 through the Research Council of Norway. The Associate Editor for this paper was M. Zhou. (Corresponding author: Rong Yu.)

J. Kang, R. Yu, and X. Huang are with the School of Automation, Guangdong University of Technology, Guangzhou 510006, China (e-mail: kjwx886@163.com; yurong@ieee.org; huangxu_min@163.com).

Y. Zhang is with the Department of Informatics, University of Oslo, 0317 Oslo, Norway, and also with the Simula Research Laboratory, 1325 Oslo, Norway (e-mail: yanzhang@ieee.org).

Color versions of one or more of the figures in this paper are available online at http://ieeexplore.ieee.org.

Digital Object Identifier 10.1109/TITS.2017.2764095
Due to the requirements of small-scale and low-cost hardware, a single vehicle generally has limited computing and storage capability to support many emerging applications, e.g., autonomous driving. Recently, mobile cloud computing has been integrated into $\mathrm{IoV}$, thus cloud-assisted $\mathrm{IoV}$ is proposed [2]. However, with mobile data traffics increasing, communications between remote cloud and vehicles easily give rise to big round-trip delay as well as high energy consumption [3]. Besides, decision making is executed in the remote cloud and far away from underlying vehicles. The cloud-assisted IoV rarely supports awareness during service provision.

We are motivated to exploit fog computing in order to improve overall performance of IoV. Fog computing is complementary to cloud computing by extending computing and storage capabilities to network edge, which enables localized decision making and low-latency communications. Previous work has integrated fog computing into vehicular networks for supporting mobility [4]. We consider that fog computing can also bring new insights and high potential to IoV, leading to a new paradigm named Fog computing supported IoV (F-IoV). In F-IoV, there exist lightweight but ubiquitous communication and computation resources. The seamless radio coverage provides high-rate and stable connections for vehicles. Supported by sufficient computation resources deployed in close proximity to users, surrounding information around vehicles can be conveniently obtained. This is of benefit to offering more context awareness (e.g., location awareness) to improve qualityof-service of vehicular applications. Moreover, the localized data processing in F-IoV saves backbone bandwidth and reduces energy consumption.

Although there are great advantages in F-IoV, security and privacy issues cannot be neglected for large-scale adoption. For driving safety, vehicles are required to periodically broadcast safety messages (consisting of current positions, speeds, accelerations and so on) to vehicle neighbors. These messages increase the awareness of vehicles about their neighbors' whereabouts and warn drivers of dangerous situations. But the safety messages also pose potential threats to location privacy of the vehicles [5]. To address this problem, real identities of the vehicles are normally replaced by pseudonyms [6]. Vehicles frequently change pseudonyms to avoid being continuously tracked. However, with ever-growing number of vehicles, pseudonym management including pseudonym generation, distribution and revocation poses a great challenge in F-IoV. 
By utilizing fog computing, pseudonym management can be significantly improved, where the capability of pseudonym management is shifted to network edge. More specifically, multiple local authorities, that locally manage their own pseudonyms, are deployed in close proximity of vehicles for decentralized pseudonym management. Thus pseudonyms are generated, distributed and revoked at the wide network edge instead of traditional centralized way for balancing management workloads in F-IoV. These local authorities are much closer to vehicles and can distribute new pseudonyms to the vehicles in time. Besides, to enhance location privacy, vehicles may choose to change pseudonyms according to real-time context information at hotspots. The context information is that how many vehicles change pseudonyms simultaneously [7]. In F-IoV, the local authorities deployed at the network edge are easy to obtain context information, and assist vehicles to change pseudonyms with contextual awareness.

In this paper, we propose a Privacy-Preserved Pseudonym $\left(P^{3}\right)$ scheme in F-IoV, and introduce specialized fogs named pseudonym fogs, which are deployed in different regions to directly manage pseudonyms for passing vehicles. The geo-distributed pseudonym fogs consist of a group of fog infrastructures, whose computing resources are gathered and virtualized to establish a local fog in a region. There exists a local authority in each pseudonym fog. According to context information provided by the local authorities, the vehicles carry out context-aware pseudonym changing in F-IoV. The contributions in this paper are summarized as follows.

- We present a three-layer architecture and specifical pseudonym fogs for pseudonym management in F-IoV.

- We propose decentralized and underlying pseudonym management communication protocols to efficiently manage pseudonyms for privacy preservation in F-IoV.

- A context-aware pseudonym changing game is formulated according to a metric named vehicle-side entropy for enhancing location privacy.

\section{RELATED WORK}

For driving safety, vehicles have to broadcast periodical safety messages including 6-tuple information \{ID, Location, Velocity, Direction, Content, Time $\}$. For privacy protection, vehicles should use pseudonyms instead of their real identities. However, under a consecutive adversary tracking, pseudonyms are still vulnerable if vehicles keep using identical pseudonyms for a long time. To address this problem, previous work has proposed three major types of schemes: 1) Mix-zone [8], 2) group signature [9], and 3) silent period [10].

A Mix-zone for vehicles to change their pseudonyms typically constructs in a road intersection with multiple vehicles [8]. The vehicles change their pseudonyms and separately depart from different exits, which achieves the unlinkability of pseudonyms. The parking lots can naturally be assigned as Mix-zones in [11]. But the mix-zone schemes are limited by the number of vehicles appearing at the pseudonyms changing occasions, which may not perform very well in the places of few vehicles or low traffic [6].

For the group signature scheme, a vehicle joins a group and signs safety messages using a group identity for location

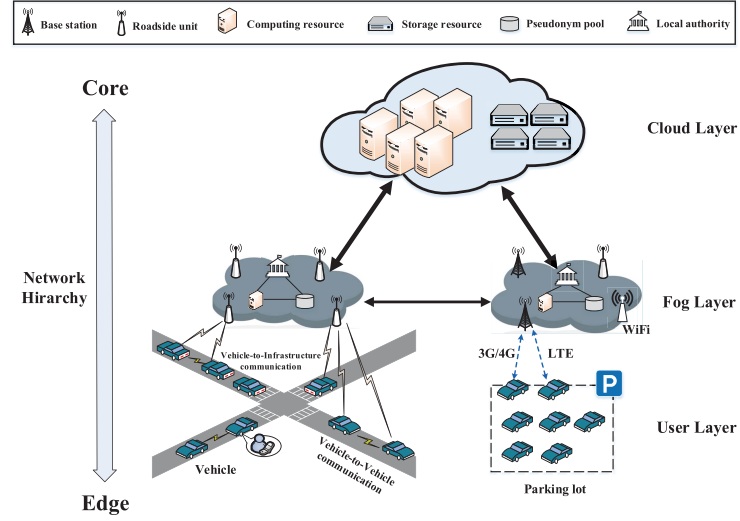

Fig. 1. Hierarchical architecture for pseudonym management.

privacy protection. Only the trust group leader knows about true identities of vehicles, and has the right to track down any group members if necessary. However, the group signature scheme is restricted by the group size [6]. A large-scale group has low efficiency in managing the signatures while a small group is weak in preserving privacy.

For the silent period scheme, a target vehicle entering a region of interest initially broadcasts safety messages, then keeps silent and updates its pseudonym for a random silent period during moving from locations $L_{1}$ to $L_{2}$, and finally broadcasts in $L_{2}$. Meanwhile, if a neighboring vehicle updates pseudonyms from locations $L_{3}$ to $L_{4}$, the adversary may be misled to treat the neighboring vehicle as the target. However, it is still possible for the adversary to track the target by inferring spatiotemporal relationship of vehicles [12].

As the vehicles periodically change pseudonyms in IoV for privacy preservation, the strategies for vehicles to obtain new pseudonyms can be broadly categorized into two groups. I) To request as many pseudonyms as possible at a time. For example, a vehicle in [12] carries about 48830 pseudonyms each year to ensure anonymity. This method, however, increases the demand on storage. Once the system revokes the pseudonyms of a malicious vehicle, it takes a long time to broadcast the revocation list resulting in higher cost and information redundancy [13]. II) To request only a few pseudonyms from a local manager, whose pseudonyms come from a remote data center (e.g., a cloud center). The vehicles repeat the aforementioned process whenever pseudonyms are needed [6]. But it is not green and efficient enough for emerging vehicular paradigms due to high communication overhead [14] between vehicles and remote data center. Therefore, it is significant to design an effective pseudonym system with context awareness, on-demand generation and timely distribution to manage pseudonyms in the new vehicular paradigms, such as F-IoV.

\section{A Hierarchical Architecture for PSEUdONYM MANAGEMENT}

\section{A. Overview of Hierarchical Architecture}

Fig. 1 shows a hierarchical architecture for decentralized pseudonym management in F-IoV. This architecture consists of three layers: cloud layer, fog layer and user layer. In the user 


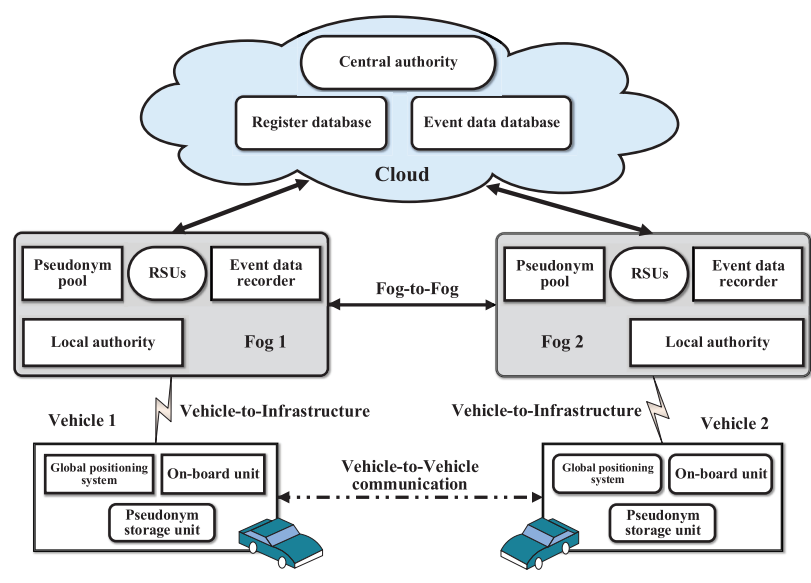

Fig. 2. Security model of pseudonym management.

layer, vehicles communicate with each other through vehicleto-vehicle communication, and connect to the fog infrastructures for requesting new pseudonyms. In the fog layer, the fog infrastructures are located at the network edge where fog environment provides cloud-like service to vehicles [15]. The fog devices can communicate with other entities through wired and wireless ways. In a certain region, the fog devices in the fog layer are combined to form a pseudonym fog. Each pseudonym fog manages and allocates pseudonym resource to nearby vehicles for location privacy protection. Compared with the fog layer, the clouds in the cloud layer (the core of the network) execute long-term and complex tasks by rich computing and storage resources. More details about the three layers are described as follows.

\section{B. Security Model in the Hierarchical Architecture}

1) Cloud Layer: There exists a central authority with the highest priority to manage pseudonyms in F-IoV, which is generally undertaken by an ITS department of government. The central authority equipped with tamper-resistant hardware is deployed in a remote cloud, which can utilize sufficient cloud resources to defend against potential attacks. Thus, the central authority is a fully trusted without being compromised [6], [16]. Before driving on the roads, vehicles should register identity-related information, which can be used for uniquely identifying the vehicle and the owner. The central authority generates and distributes public, private keys and digital certificates to all vehicles after registration. These registration information is stored into a register database in the remote cloud, as shown in Fig. 2. The central authority is trusted to manage initial security parameters and keys of vehicles. The global pseudonym usage information of all vehicles (e.g., the used and using pseudonyms) is also uploaded and stored into the register database. There is also an event database in the remote cloud. The event database is to record normal and violation events happened in F-IoV for responsibility investigation. More details are illustrated in Section IV.

2) Fog Layer: In F-IoV, nearby network infrastructures deployed at the network edge (e.g., roadside units and enhanced base stations) connect with each other to form pseudonym fogs. In the network infrastructures, there exist dedicated local fog servers that connect to the Internet by wired way, and provide radio interfaces for vehicles to access computing and storage resources. These fog servers utilize network function virtualization technology to highly virtualize physical resources in the fog infrastructures, and thus establish virtual machines for computing instances. Besides, for flexible resource allocation among the fog servers, software-defined networking technology is used to dynamically create, migrate, offload and destroy the virtual machines according to different network states. Based on these technologies, fog layer in F-IoV is implemented in practical scenarios.

In the fog layer, each pseudonym fog mainly consists of four components including a pseudonym pool, fog devices (e.g., roadside units), a local authority, and an event data recorder in F-IoV. The pseudonym pool locally generates and stores pseudonyms in F-IoV. The RSUs combine with each other to provide edge resources. The local authority consists of three parts: issuance department, serving department and revocation department. The issuance department distributes new pseudonyms generated by the pseudonym pool to requested vehicles. The serving department builds a list to record pseudonym usage of local vehicles. With the help of this list, we can match every pseudonym with the corresponding vehicle. The event data record not only records all the misconduct events of vehicles or RSUs in a local pseudonym fog, but also monitors adjacent pseudonym fogs and other local authorities for mutual supervision. Then the event data recorder generates a blacklist and uploads it to the event data database in remote cloud for revealing true identity and investigating responsibility of illegal vehicles. The pseudonym fogs communicate with each other through wired or wireless communication technologies. The local authority can transmit authentication information of vehicles to other local authorities, and thus authenticates the legality of pseudonyms by the pseudonym fogs which generate the pseudonyms.

3) User Layer: Vehicles are equipped with on-board units, wireless communication devices, a global positioning system, and a pseudonym storage unit. The pseudonym storage unit is used to store pseudonyms issued by local pseudonym fog and corresponding public and private keys. From the perspective of system, there are many global social hotspots, where a number of vehicles meet at a certain time. For example, a road intersection of a busy street is a typical global social hotspot. It is beneficial for vehicles to regard the social hotspots as natural mix-zones, and collectively change the pseudonyms at the spots for higher levels of location privacy [5], [11]. Vehicles in these hotspots may participate in a pseudonym changing game to determine whether to change their pseudonyms when arriving at the hotspots. For efficient pseudonym changing, context information, that how many other vehicles want to participate in changing pseudonym synchronically, is the key point for rational decision making of vehicles. In F-IoV, the local authorities collect the information and broadcast the information to vehicles in the social hotspots. Through realtime intersection with the local authorities, the vehicles make 
various decisions of pseudonym changing according to the dynamic context information. When the context information is changeless, the decisions also are stable.

\section{Advantages of the Hierarchical Architecture}

Compared with most of the existing pseudonym management architectures [18], [19], our proposed architecture shifts the capabilities of pseudonym management to network edge. Management workloads in the network are balanced and spread to different pseudonym fogs. Decentralized pseudonym management is localized by deploying local authorities in close proximity to vehicles. It is convenient for the local authorities to quickly respond to pseudonym requests and distribute new pseudonyms [4]. Gao et al. [3] carry out tests to show that edge data centers (e.g., cloudlets) improve the response time and decrease energy consumption of mobile devices (51\% and $42 \%$, respectively) in comparison to centralized clouds. Similarly, the edge deployment of multiple local authorities reduces energy consumption of communications for pseudonym management. Besides, the local authorities also collect context information to assist vehicles to change the pseudonyms in social hotspots with contextual awareness.

More details about the advantages of our architecture are summarized as follows:

- Reduced management overheads: In the network, total workload of pseudonym management is balanced in geo-distributed pseudonym fogs. The local authorities in the pseudonym fogs enable decentralized pseudonym management in the underlying layers. "short-fat" communications are supported when the proximal local authorities interact with bypassing vehicles. This significantly reduces management overheads, owing to bandwidth reduction and power decrease.

- Timely pseudonym distribution: The edge deployment of multiple local authorities is convenient for distributing new pseudonyms promptly. Pseudonym generation and distribution are undertaken in a distributed manner. This lessens congestion delay in case that a large number of simultaneous pseudonyms requests incur. The pseudonym requests are detected and locally processed with less response time and round-trip delay. Overall, the requesting vehicles are able to obtain new pseudonyms in time.

- Context-aware pseudonym changing: The local authorities broadcast necessary context information for vehicles in the social hotspots. The vehicles choose whether to change pseudonyms based on the context information. For vehicles, the context-aware pseudonym changing strategies can be performed well with the real-time interactions of the local authorities. Due to the assistance of local authorities, more vehicles can participate in synchronized pseudonym changing. As a result, the achieved location privacy is improved.

\section{Privacy-Preservation Pseudonym Scheme}

\section{A. A Brief Overview of the $P^{3}$ Scheme}

In the $P^{3}$ scheme, we consider that all local authorities in pseudonym fogs locally manage their own distributed pseudonyms. Each serving department in its local authority generates a serving list to record the pseudonyms of vehicles under management. The serving lists in different local authorities are periodically uploaded to the register database of the central authority, and thus form a global tracking table in the event data database. In F-IoV, even a vehicle with pseudonyms distributed by a local authority leaves from the local region and enters the next region, the vehicle still be managed by the previous local authority till it requests new pseudonyms from other authorities. The cooperation among these two local authorities is necessary for updating serving lists.

In this paper, every local authority manages its locally generated pseudonyms because of the following advantages. I) The private information of every vehicle is only recorded in corresponding local authority instead of sharing among several authorities to decrease the probability of privacy leakage. II) If a vehicle is compromised, the local authority with the whole information of the vehicle can timely revoke corresponding pseudonyms of the vehicle. This clearly avoids superfluous searching for vehicles with a higher efficiency among local authorities. III) We consider that most vehicles usually travel within a region, such as a city [21]. All local authorities are connected by a wired network to improve information transmission. Therefore, cross-region pseudonym management among local authorities can be performed in a decentralized and efficient manner with acceptable overheads.

A vehicle $v_{i}$ periodically broadcasts safety messages with its own pseudonyms $\left\{P I D_{i}^{k}\right\}_{k=1}^{w}$ for safe driving. When $v_{i}$ has no sufficient pseudonyms, it requests pseudonyms from the nearest local authority according to current location. After local pseudonym requesting operation, $v_{i}$ receives the pseudonyms and changes them in the local region. Consequently, local pseudonym changing operation is triggered. When $v_{i}$ travels to other regions and hold pseudonyms issued by previous local authority. With the cooperation among local authorities, $v_{i}$ can continue to broadcast safety messages using the pseudonyms by a cross-region pseudonym changing protocol. When $v_{i}$ runs out of existing pseudonyms, $v_{i}$ requests new pseudonyms from a new local authority, and a cross-region pseudonym requesting protocol will be performed.

To implement the $P^{3}$ scheme, two key mechanisms are devised as below. The main symbols used in this scheme are listed in Table I.

- Pseudonym mechanism: In this paper, a vehicle $v_{i}$ requests $w$ new pseudonyms on demand. The number of requesting pseudonyms (e.g., 40 pseudonyms one day) is depended by vehicles' driving plans [12], [22]. Efficient pseudonym changing can avoid being consecutively tracking. In social hotspots, $v_{i}$ changes pseudonym according to current context information. Between two social hotspots, vehicles can periodically change pseudonyms during driving, e.g., once every 12 minutes.

- Encryption and authentication: To protect wireless communication security and exclude illegal vehicles, the $P^{3}$ scheme uses restrict encryption and authentication mechanisms. For each vehicle $v_{i}$, there are three sets of public and private keys and certificates for real identity, safety message broadcasting, and context-aware 
TABLE I

SYMBOLS USED IN THIS PAPER

\begin{tabular}{c|l}
\hline Notation & Description \\
\hline$v_{i}$ & The $i^{t h}$ vehicle. \\
$R S U_{j}$ & The $j^{t h}$ RSU. \\
$P I D_{i}^{k}$ & The $k^{t h}$ pseudonym of vehicle $i$. Each vehicle has $w$ \\
pseudonyms, $\left\{P I D_{i}^{k}\right\}_{k=1}^{w}=\left\{P I D_{i}\right\}$. \\
$P K_{i}, S K_{i}$ & Public and private key pair of vehicle $v_{i}$. \\
$C e r t_{i}$ & Certificate of vehicle $v_{i}$. \\
$L C_{j}$ & The $j^{t h}$ local pseudonym fog. \\
$L A_{j}$ & The local authority of $L C_{j}$. \\
$\{x\}$ & A set with element $x$. \\
$i \rightarrow j$ & Entity $i$ sends a message to entity $j$. \\
$x \| y$ & Element $x$ concatenates to $y$. \\
$E_{P K_{i}}(m)$ & Encryption of message $m$ with public key of $i$. \\
$E_{S K_{i}}(m)$ & Encryption of message $m$ with private key of $i$. \\
$S i g n_{S K_{i}}(m)$ & Digital signature on $m$ with private key of $i$. \\
$G I D_{c}$ & The $c^{t h}$ group including vehicles wanted to change \\
CA & pseudonyms. \\
$T s$ & Central authority \\
Tw & Timestamp of event. \\
\hline
\end{tabular}

pseudonym changing, respectively. Specifically, $\left\{P K_{i}\right.$, $\left.S K_{i}, \mathrm{Cert}_{i}\right\}$ are used in vehicle-to-infrastructure communications instead of the vehicle's real identity; $\left\{P I D_{i}^{k}\right.$, $\left.S K_{P I D_{i}^{k}}, \operatorname{Cert}_{P I D_{i}^{k}}\right\}$ protect true identity when the vehicle broadcasts safety messages; $\left\{G I D_{c}, S K_{G I D_{c}}\right.$, $\left.\operatorname{Cert}_{G I D_{c}}\right\}$ are to send requests and responses before pseudonym changing.

\section{B. Details About Key Operations}

1) System Initialization and Key Generation: We utilize an efficient Boneh-Boyen short signature scheme for system initialization and key generation. $v_{i}$ with true identity $I D_{i}$ first joins the F-IoV. Then $v_{i}$ obtains its public/privacy key pair and certificate (denoted as $P K_{i}, S K_{i}$, and $\mathrm{Cert}_{i}$ ) from the central authority in remote cloud. The central authority notifies the nearest pseudonym fog (e.g., $L C_{j}$ ) of $v_{i}$ to distribute a set of $w$ pseudonyms $\left\{P I D_{i}^{k}\right\}_{k=1}^{w}$ to $v_{i}$. These pseudonyms are attached with corresponding public/private key pair and certificate, i.e., $P I D_{i}^{k}, S K_{P I D_{i}^{k}}$, and $\operatorname{Cert}_{P I D_{i}^{k}}$. The central authority generates a tracking table $\left\{I D_{i}, P K_{i}^{i}, \operatorname{Cert}_{i}, L C_{j}\right.$, $\left\{P I D_{i}^{k}\right\}_{k=1}^{w}$, and $\left.\left\{\operatorname{Cert}_{P I D_{i}^{k}}\right\}_{k=1}^{w}\right\}$ in the event data database. Then the central authority distributes a tracking list $\left\{P K_{i}\right.$, $\operatorname{Cert}_{i}, L C_{j},\left\{P I D_{i}^{k}\right\}_{k=1}^{w}$, and $\left.\left\{\operatorname{Cert}_{P I D_{i}^{k}}\right\}_{k=1}^{w}\right\}$ to all RSUs after encryption using the RSUs' public keys. We consider that all the RSUs are trusted in line with the assumptions usually accepted for IoV [23], [24]. All RSUs are linked with each other for well cooperation and mutual supervision. The RSUs with misconduct will be accused and held accountable in a bounded time period.

2) Basic Operations for Pseudonym Management: When $v_{i}$ drives in a local region, $v_{i}$ periodically broadcasts safety messages with pseudonyms $\left\{P I D_{i}^{n}\right\}_{n=1}^{\alpha}$ every $300 \mathrm{~ms}$ [11]. $v_{i}$ changes pseudonyms to prevent continuous tracking from a potential adversary during driving. In order to ensure liability of the message originator and safety of message receivers, $v_{i}$ signs its safety messages with a time-stamp to ensure message freshness, and includes a pseudonym certificate to enable verification [5]. $v_{i}$ also receives safety messages from nearby neighbors, and verifies these messages according to attached signatures and time-stamps. Before running out of all pseudonyms, $v_{i}$ will execute local or cross-region pseudonym requesting protocols according to its current location. Similarly, if $v_{i}$ wants to change pseudonyms, $v_{i}$ will perform local or cross-region pseudonym changing protocols. More details about these operations are described in Protocol 1.

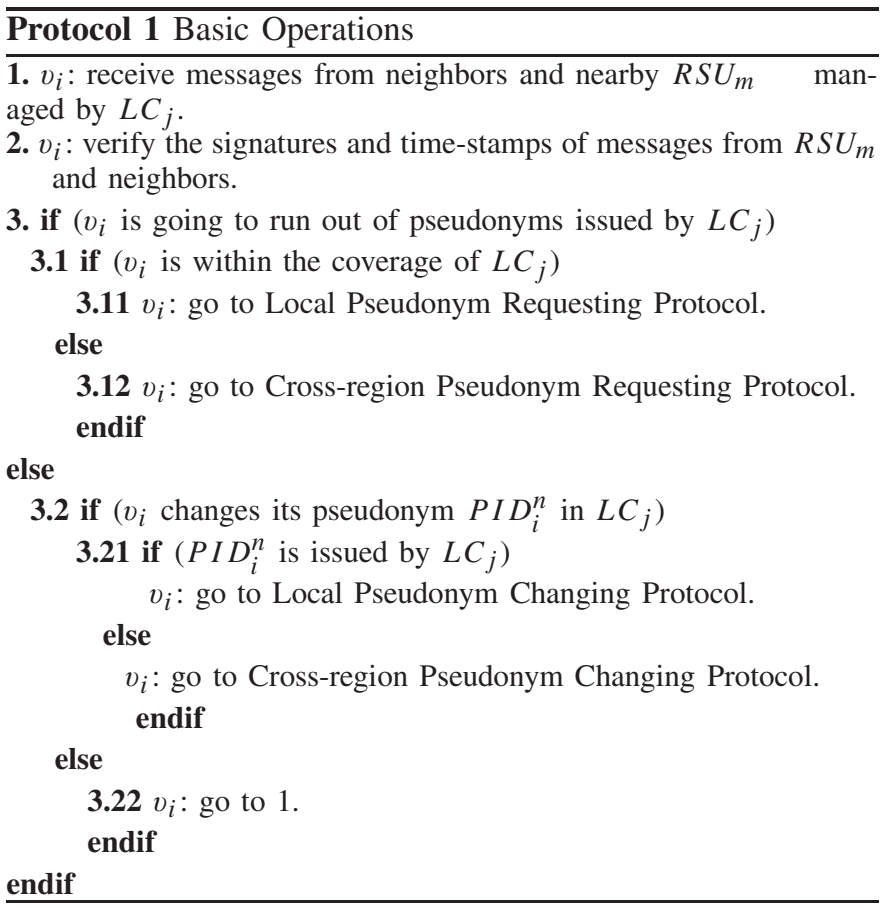

3) Local Pseudonym Requesting: Before running out of all pseudonyms, $v_{i}$ has to request new pseudonyms for privacy protection. $v_{i}$ sends a pseudonym request including the number of requesting pseudonyms, current location, public key of pseudonym being used (i.e., $P I D_{i}^{n}$ ), public key for IoV communication, and corresponding certificates $\left(\operatorname{Cert}_{i}\right.$ and $\left.\operatorname{Cert}_{P I D_{i}^{k}}\right)$ to a local authority $\left(L A_{j}\right)$ after encryption. $L A_{j}$ verifies the legitimacy of $v_{i}$ 's information. If $v_{i}$ is in the coverage of $L A_{j}$ and is legitimate, $L A_{j}$ will send a set of new pseudonyms $\left\{P I D_{i}^{k}\right\}$ with corresponding public/ private keys, certificates attached with expiry time to $v_{i}$. After verification, $v_{i}$ stores these new pseudonyms and sends back a confirmation to $L A_{j}$. Then $L A_{j}$ generates and updates a record about $v_{i}$ 's, consisting of its communication identity, requesting pseudonyms and the number, corresponding certificates, the currently using pseudonym, the used local pseudonyms, timestamp and so on. By being uploaded to the central authority, the newest record is put into the tracking table for establishing the mapping relationship of $v_{i}$. More details about local pseudonym requesting are presented in Protocol 2.

4) Context-Aware Pseudonym Changing Grouping: For the vehicles, the pseudonym changing is based on the total 

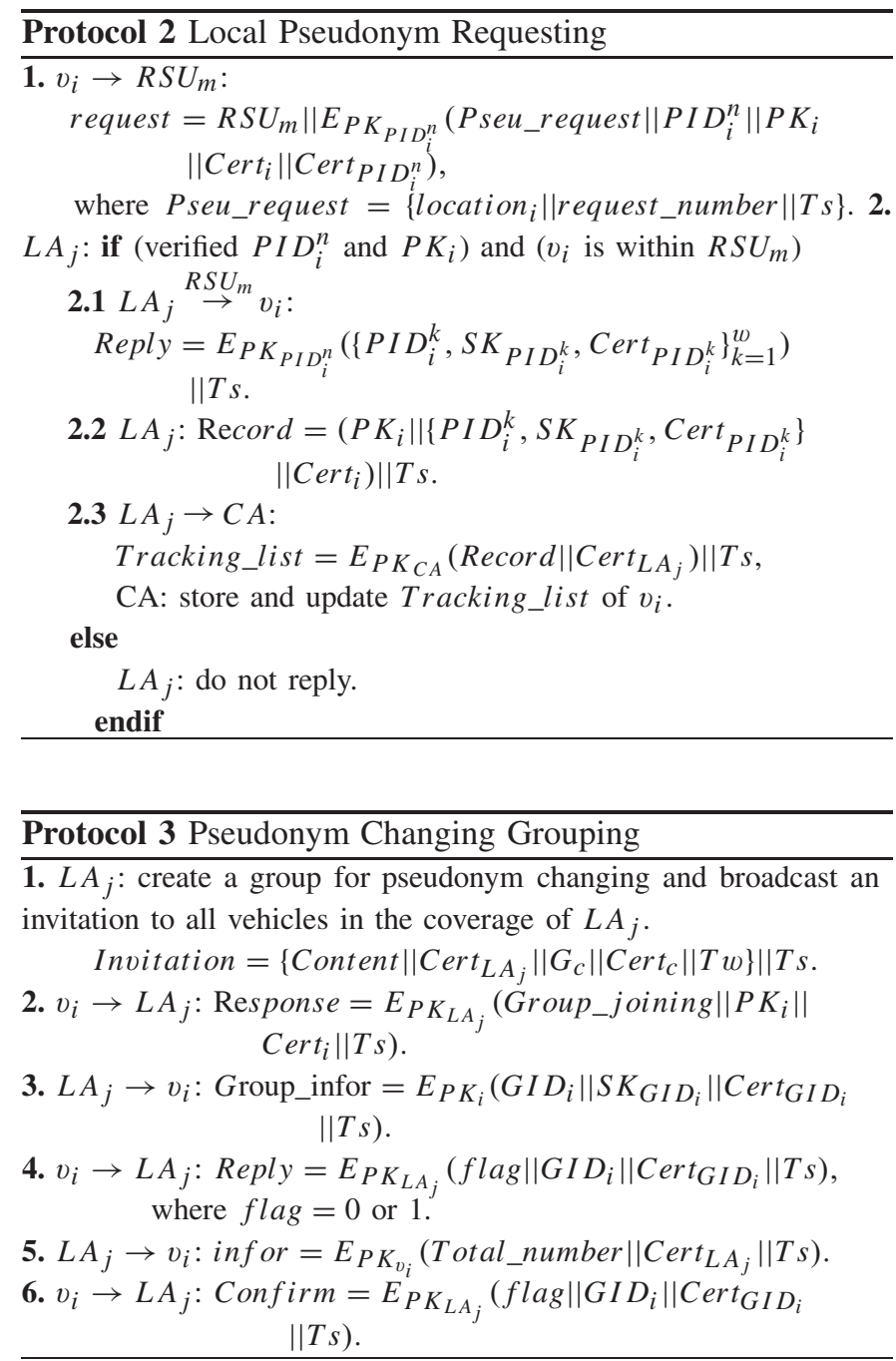

number of vehicles changing pseudonyms simultaneously. More details about group size of pseudonym changing are given in Section $\mathrm{V}-\mathrm{C}$. The $L A_{j}$ works as a group manager and creates a group $G_{c}$ for context-aware pseudonym changing, and then broadcasts an invitation to all vehicles as shown in Protocol 3. The invitation includes group-related information (i.e., $G_{c}$ and $\mathrm{Cert}_{c}$ ), time window for pseudonym changing $T w$, and invitation content and so on. The vehicles in the coverage of $L A_{j}$, that want to join $G_{c}$, verify the invitation and send back their responses to $L A_{j}$ with identityrelated information. Here, we take $v_{i}$ as an example. After $L A_{j}$ verifies the response from $v_{i}, L A_{j}$ sends the group identity-related information to $v_{i}$. If $v_{i}$ wants to change its pseudonym, $v_{i}$ sends a reply with group identity information and a flag ("1" or "0") indicated willingness of pseudonym changing to $L A_{j}$. Here, " 1 " is to join the synchronized pseudonym changing, while " 0 " is not. After a certain time, $L A_{j}$ calculates the total number of those vehicles that determine to join, and sends this context information to all the vehicles. Then $v_{i}$ decides dynamically whether to change its pseudonym at a specific time window according to the context information. After that, $L A_{j}$ collects their responses, updates the context information and broadcasts them once again and till the context information is changeless. Then the vehicles that finally determine to join the synchronized pseudonym changing proper to change their pseudonym collectively. Thus, the grouping of context-aware pseudonym changing is finished.

5) Local Pseudonym Changing: If $v_{i}$ using PID $D_{i}^{k}$ wants to change its pseudonym, it will send a message shown in Protocol 4 to $L A_{j}$ after encryption. The message includes the current and the next pseudonym $P I D_{i}^{k+1}$, corresponding certificate, identity $P K_{i}$, its signature and timestamp. Here, $P I D_{i}^{k+1} \in\left\{P I D_{i}^{k}\right\}_{k=1}^{w}$. $L A_{j}$ verifies the validity of received information about $v_{i}$. The verification is to authenticate the legal pseudonym certificates and individual signature. After the authentication, the local authority confirms that it is a local vehicle and updates the serving record about $v_{i} . L A_{j}$ sends a reply to $v_{i}$ to notify the confirmation of pseudonym changing. $L A_{j}$ puts all the records about local vehicles into a serving list. Then $L A_{j}$ periodically sends the newest serving list to the $\mathrm{CA}$ for updating the tracking table in the event database.

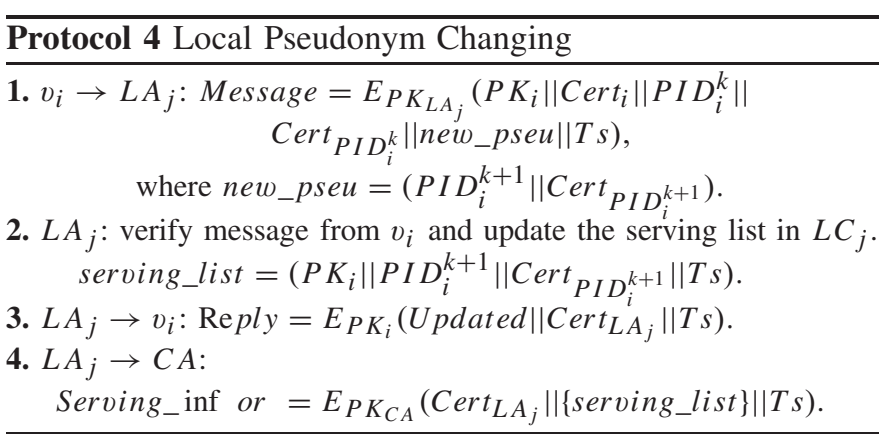

6) Cross-Region Pseudonym Requesting: If $v_{i}$ is using the last pseudonym $P I D_{i}^{w-1}$ distributed by $L A_{j}$ in another area (managed by $L A_{m}$ ), $v_{i}$ will send a pseudonym request to $L A_{m}$. This pseudonym request includes the identity information about $v_{i}$ and the current pseudonym. Here, the pseudonym request is request $=$

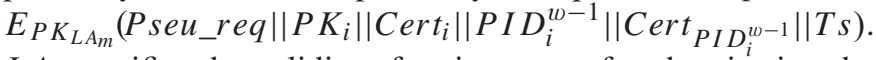
$L A_{m}$ verifies the validity of $v_{i}$ in terms of authenticating the certificate of the old pseudonym and its signature. After the authentication, $L A_{m}$ communicates with $L A_{j}$ for transmitting a record removing notification about $v_{i} . L A_{j}$ stops updating the record about $v_{i}$. At the same time, $L A_{m}$ begins to record $v_{i}$ and distributes $v_{i}$ a set of new pseudonyms. The process and operations of pseudonym distribution are similar to that of local pseudonym requesting.

7) Cross-Region Pseudonym Changing: When $v_{i}$ is using $\left\{P I D_{i}^{k}\right\}$ (issued by $L A_{j}$ ) in the coverage of $L A_{m}, v_{i}$ wants to change pseudonym and sends a cross-region request to $L A_{m}$. The message is the same as that in the local pseudonym changing operation. $L A_{m}$ receives the request from $v_{i}$ and also verifies its validity. By authentication, $L A_{m}$ knows that it is a vehicle managed by $L A_{j} . L A_{m}$ transmits a record updating notification about $v_{i}$ to $L A_{j}$ after encryption with $L A_{j}$ 's public key. Then $L A_{j}$ verifies this notification and updates the record on $v_{i}$ in the serving list.

8) Pseudonym Revocation: In $P^{3}$ scheme, any violation of vehicles will be monitored and accused by neighboring 
vehicles or fog infrastructures. For example, if a compromised vehicle $v_{k}$ using $P I D_{k}$ is detected by $v_{i}$, $v_{i}$ will record the violation actions of $v_{k}$, and reports to nearby local authority $L A_{j}$. There are vital evidences in the report including the type of violation of $v_{k}$, current pseudonym certificates of $v_{i}\left(\operatorname{Cert}_{P I D_{i}}\right)$ and $v_{k}$, and an event signature of $v_{i}$. Here, Report $=E_{P K_{L A}}$

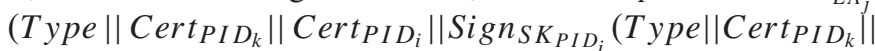
$\left.\left.\operatorname{Cert}_{P I D_{i}} \| T s\right) \| T s\right)$. If $v_{k}$ has joined a group $G_{k}$, it will integrate group ID $G I D_{k}$, and the certificate $\operatorname{Cert}_{G I D_{k}}$ into the report. After receiving the report, $L A_{j}$ will check the validity of the report as well as the identity of $v_{i}$, and stores the report in an event data recorder. Then the report is forwarded to the central authority. The central authority will validate the report and reveal the true identity of $v_{k}$ by the tracking table in central authority. If the violation is confirmed, central authority will add $v_{k}$ into its blacklist and spread the information to the RSUs nearby the vehicles.

\section{A Context-Aware Pseudonym Changing Game}

\section{A. Threat Model}

In F-IoV, an eavesdropper may track a target vehicle by leveraging these periodical safety message broadcasts [11]. In practice, the adversary may deploy some infrastructures with eavesdropping devices in the network. We consider that a global passive adversary can locate and track any vehicle in a region-of-interest by eavesdropping the safety messages [5]. But generation and distribution process of pseudonyms and keys cannot be altered or controlled by the adversary.

There exist other approaches for an adversary to track a target vehicle. For example, a video-based approach using traffic monitoring cameras is able to visually identify the target. However, these approaches require significant efforts like expensive cameras with sufficiently high resolution to track even a single target vehicle. The adversary has to undertake overwhelming cost of the entire system. In this paper, we consider the adversary using the radio-based eavesdropping, which involves only a moderate system expense.

\section{B. Vehicle-Side Pseudonym Entropy}

The global passive adversary eavesdrops safety message broadcasts in the entire network to track the target vehicles. Vehicles use multiple pseudonyms and change the pseudonyms to broadcast safety messages for reducing long-term linkability. Here, the level of location privacy is quantitatively evaluated by a metric named pseudonym entropy [5], [7]. The entropy metric evaluates the uncertainty in mapping different pseudonyms to real identities of vehicles from the perspective of the adversary. Consider a social hotspot (e.g., an intersection and a parking lot) where a set of vehicles, denoted by $V=$ $\left\{v_{1}, v_{2}, \cdots, v_{K}\right\}$, will change pseudonyms collectively. Let $p_{i}$ represent the successful tracking probability of vehicle $v_{i}$ after pseudonyms changing. If the tracking probabilities of all the vehicles in group $V$ satisfy a uniform probability distribution, the maximal entropy achieved in $V$ is given by

$$
H_{V}^{\max }=-\sum_{i=1}^{k} p_{i} \log _{2} p_{i}=\log _{2} K .
$$

Similar to most existing work, we use the upper bound as an approximation to evaluate the location privacy of vehicles in terms of entropy. Clearly, the achieved entropy depends on the number of vehicles changing pseudonyms simultaneously. At a social hotspot, a vehicle, that wants to change its pseudonym, initiates pseudonym changing using the Protocol 3 in Section IV-B. Vehicles in proximity receive the invitations from the group manager in the Protocol 3, and enter a time window during which the vehicles decide whether to change their pseudonyms or not according to context information. At the end of the time window, those vehicles that are willing to cooperate in synchronized pseudonym changing change their pseudonyms simultaneously.

Similar to that in [7], we propose a vehicle-side entropy metric, where each vehicle locally estimates its location privacy level over time. The continuous observation of the adversary leads to entropy loss for vehicles. When the entropy is decreased to 0 , the vehicle may be tracked. To protect the location privacy, each vehicle consciously estimates its potential entropy. The vehicle decides that whether to change its pseudonym when arriving at the hotspots.

Vehicles can estimate their pseudonym entropy values at each time slot after pseudonym changing. In this paper, the vehicle-side entropy for $v_{i}$ is expressed as

$$
\hat{H}_{i}=H_{c, i}^{l}-\hat{\theta} \Delta t
$$

where $H_{c, i}^{l}$ indicates the entropy obtained from the last time of pseudonym changing. $\Delta t$ is the time interval from the last time of pseudonym changing to current time. $\hat{\theta}$ is an estimation value of entropy loss, which measures tracking power of the adversary from the perspective of $v_{i}$. With increasing $\hat{\theta}$, the vehicle tends to change pseudonyms more frequently. The long-term and complex computation of parameter estimation (e.g., $\hat{\theta}$ ) is conducted in the remote cloud. Finally, the system acquires the corresponding mean value and standard deviation about $\hat{\theta}$, denoted as $\mu$ and $\sigma$, respectively.

\section{Entropy-Enhanced Pseudonym Changing for $P^{3}$ Scheme}

In this paper, we formulate a context-aware pseudonym changing game in F-IoV. Vehicles are willing to change pseudonyms at hotspots with more vehicles changing pseudonyms simultaneously for higher privacy level. But the vehicles may not change pseudonyms at every hotspot, especially when they have achieved high-level location privacy at the previous hotspots.

We define a game $G=(V, S, U)$ to formulate the procedure of pseudonym changing based on context information. $V$ is a set of vehicles in a hotspot. $S$ is the set of strategies in the game. For a vehicle in $V, v_{i}$, it chooses to change $(C)$ or maintain $(M)$ the current pseudonym. $U$ is the set of utility functions. For $v_{i}$, its utility function is expressed by

$$
\begin{aligned}
u_{i}(C, n) & =w_{c} H_{c, i}(n)-c, \\
u_{i}(M) & =w_{m} \bar{H}_{i} .
\end{aligned}
$$

$u_{i}(C, n)$ is the obtaining utility when $v_{i}$ changes the pseudonyms with $(n-1)$ followers, while $u_{i}(M)$ is the utility of maintaining the old pseudonym. Here, $c$ is the cost of changing 
pseudonyms. $w_{c}$ is the willingness of changing the pseudonym and can be calculated by

$$
w_{c}=\left(1+\sigma \Delta t / \bar{H}_{i}\right) .
$$

This means that $w_{c}$ is related with the coefficient of variation of $\hat{H}_{i}$. The coefficient of variation is the ratio between the standard deviation $\sigma \Delta t$ and the mean value $\bar{H}_{i}=H_{c, i}^{l}-\mu \Delta t$. The coefficient of variation is used to measure whether there exists a potentially big variation in the estimating process. Here, when the variation is bigger, the vehicle has less knowledge of the adversary and it is of higher willingness to change the pseudonym for ensuring high enough privacy levels. At the same time, its willingness of maintaining the old pseudonym (denoted as $w_{m}$ ) is lowered. Besides, we consider that vehicles are conservative players in the game and they always tend to change the pseudonyms as all of them know the existence of the adversary. So we set that $w_{m} \leq w_{c}$. Hence, we also define $w_{m}$ with the coefficient of variation as follows:

$$
w_{m}=\left(1-\sigma \Delta t / \bar{H}_{i}\right) \text {. }
$$

$c$ is the pseudonym changing cost and can be expressed as a causing decrease in the achieved entropy [7].

By comparing the utilities in two cases, the vehicle determines the best strategy finally. Clearly when $u_{i}(C, n)>$ $u_{i}(M)$, the vehicle will determine to act as a rational follower to join the synchronized pseudonym changing. The determination exactly depends on the context information in the game, namely, the number of all followers at this time, $n$. With the condition of $u_{i}(C, n)>u_{i}(M)$, for every vehicle, it is easy to obtain a threshold value $n_{t h}^{i}$ that instructs the consequent actions of the vehicle. In other words, when $n>n_{t h}^{i}$, the rational vehicle chooses to participate in pseudonym changing. Otherwise, it maintains the old pseudonym as $n \leq n_{t h}^{i}$.

For the vehicles, the pseudonym changing game is established among real-time interactions with local authorities. Here, we take an example that the vehicles communicate with an RSU in a road intersection to access the closest local authority. The game may consist of multiple rounds but this game can be performed well in fog environment. In F-IoV, localized decision making is supported for latency-sensitive computations and analysis. Besides, prompt communications are achieved between the local authority and vehicles, owing to low response time and round-trip delay. The detailed procedure of this game is performed as follows.

Theorem 1: For any round of the game, there always exists $n_{r-1} \geq n_{r}$.

Proof: In the first round, $n_{1} \leq n_{0}=K$. Specially, when $n_{1}=K$, the game achieves the the final result that all the players participate in the synchronized pseudonym changing. If $n_{1}<n_{0}$, it demonstrates that for some vehicles belonging to a set denoted by $A, \forall j \in A, n_{t h}^{j}>n_{0}$ is satisfied. Due to the rationality of the vehicles in $A$, they will not regret and change their strategies, from $M$ to $C$ in the second round because of $n_{2} \leq n_{1}<n_{t h}^{j}$. Besides, with the update context information $n_{1}$, which is smaller than $n_{0}$, some vehicles choosing $C$ in the first round may change their strategies to $M$ in the second round. Thus, $n_{2}$ will be less than or equal to $n_{1}$ in

\section{The procedure of the pseudonym changing game}

Step 1: The LA schedules the vehicles that arrive in a certain time window to join this game. The RSU creates a player set, $V$, including $K$ vehicles.

Step 2: Initialize $r=1$. The LA broadcasts the initialized context information $n_{r-1}=K$ to all the players in $V$ via the RSU,

Step 3: In the $r_{t h}$ round, $v_{i}$ in $V$ knows that there will be $n_{r-1}$ followers if it also participates in the pseudonym changing in this round. When $n_{r-1}>n_{t h}^{i}, v_{i}$ decides to change the pseudonym. Otherwise, $v_{i}$ maintains the old pseudonym. $v_{i}$ replies to its decision $S_{i}$ to the RSU. If $S_{i}=M$, this also means that $v_{i}$ will split from $V$ and exit from this game.

Step 4: The LA receives all the replies and counts the newest number of those vehicles that decide to change the old pseudonyms in the $r_{t h}$ round. Thus, the context information in this round, $n_{r}$, is updated.

Step 5: If $n_{r}$ is equal to $n_{r-1}$, the LA informs those vehicles that still exist in $V$ to get ready for changing their pseudonyms at the same time. The game terminates. Otherwise, perform the next round of the game, $r=r+1$. Return to Step 3 .

the second round. The similar conclusion is moved to the next round, and so on. Until when $n_{r}=n_{r-1}$, the game terminates.

Theorem 2: The game can coverage and reach to the Nash equilibrium.

Proof: Based on the Theorem 1, the size of the player set $V$ is decreasing round by round. After the limited rounds, the game reaches its convergence when the player set $V$ is unchanged. When the final size is non-zero (denote as $n^{*}$ ), an available player set $V^{*}$ is emerged to confirm vehicles that participate in the synchronized pseudonym changing. All the vehicles in this set satisfy: $\forall j \in V^{*}, n_{t h}^{j}>n^{*}$. For these vehicles, they have made the best strategies $(C)$ in the case that $u_{j}(C, n *)>u_{j}(M)$. In the situation, all the vehicles have no motivation to change their decisions and take other actions. Thus, Nash equilibrium can be achieved at the steady state, where the game is converged. Finally, the number of the vehicles participating in the synchronized pseudonym changing can be obtained by the following inequality group with respect to $k$,

$$
\left\{\begin{array}{l}
1 \leq k \leq n, \\
N[k] \leq k .
\end{array}\right.
$$

$N$ is the ascending sorted set of all the threshold values $n_{t h}^{i}$. $N[k]$ indicates the $k_{t h}$ element in the set $N$. The solution set of the above inequality group is denoted by $B$. When $B$ is not an empty set, $n^{*}$ is calculated by

$$
n^{*}=\max (B),
$$

where max $(B)$ returns the maximal element in the set $B$. The group size finally reaches to a convergency that all vehicles in the group can achieve a higher level of location privacy.

\section{Vi. Performance Evaluation}

\section{A. Security Analysis}

1) Anonymity of vehicles: The strength of location privacy preservation depends on the uncertainty (i.e., pseudonym 
entropy) in mapping pseudonym to real identities of vehicles from the perspective of a global passive adversary [8], [11]. The global passive adversary passively eavesdrops vehicles' safety messages and observes time and locations of vehicles in order to derive a possible mapping of target vehicles' pseudonyms. If there are few vehicles in the pseudonym changing place, the adversary may have high probability to track the target vehicles. In the situations with sparse vehicles, the vehicles periodically change their pseudonyms (e.g., once every 12 minutes [12]) for privacy protection. At the hotspots, the vehicles estimate their own vehicle-side pseudonym entropy to decide the time and places of pseudonym changing according to current context information. In this case, to maximize vehicle-side pseudonym entropy, the vehicles are more willing to change pseudonyms at hotspots with sufficient vehicles. It is hard for the global passive adversary to continuously track a target when the target is "mixed" with many vehicle neighbors. All these vehicles look like the target vehicles if the vehicles change their pseudonyms simultaneously at the hotspots. As a consequence, the global passive adversary will be lost in tracking a target vehicle. Compared with centralized pseudonym management, communication consumption of our scheme mainly includes moderate communication overhead during pseudonym changing game.

2) Basic Security Requirements: The $P^{3}$ scheme has favorable defense ability against many security attacks through standard cryptographic primitives including asymmetric/symmetric key-based encryption. For example, due to the encryption and authentication mechanisms, the adversary cannot open the encrypted messages by launching brute-force cryptanalytic attacks. The replay attacks would not be successful due to the usage of timestamps. Meanwhile, the adversary cannot simulate an RSU or forge the RSU messages. In order to defend against sybil attacks, we consider that when a vehicle changes its pseudonym, the old pseudonym expires and is removed from the vehicle's memory.

3) Data Integrity and Authentication: With the help of digital signatures, all entities verify the digital signature during the communication. Without the private key of the signer, any entity cannot counterfeit the digital signatures of other entities. Since a digital signature is only generated by a specific signer, any information with a digital signature can be authenticated and verified whether the signer is the sender or not. So the adversary is hard to emulate the legal vehicles. If one message is modified, the receivers can discover it during verification, which guarantees the integrity and authentication.

\section{B. Numerical Results}

We consider a vehicular fog computing scenario, where has $10 \times 10$ uniform street grids in a $20 \mathrm{~km}^{2}$ region of the central business district. The streets are two-lane roads with bidirectional traffic. There are 8 large intersections treated as social hotspots in these streets, in which each hotspot has a nearby deployed RSU. The radio coverage radius of RSUs is set to be $350 \mathrm{~m}$. The vehicles arrive at the intersections as a Poisson process [11], whose mean value $(\lambda)$ ranges from 30 to 120 vehicles per minute. The red time of traffic light in each intersection is set as 60 seconds. The rate of entropy loss $\hat{\theta}$ (entropy/minute) satisfies a normal distributed characteristic: $\hat{\theta} \sim N\left(0.1, \sigma^{2}\right)$. The cost of changing pseudonyms $(c)$ takes a value from the set $[0.3,0.5,0.7]$ [7]. The observation time is 120 minutes. Besides, we use same parameters on cryptographic operations and transmission delays in [18]. More specifically, the time of main cryptographic operations is listed as follows: $1.86 \mathrm{~ms}$ for asymmetric encryption, while $0.94 \mathrm{~ms}$ for decryption; $0.93 \mathrm{~ms}$ and $1.11 \mathrm{~ms}$ for signature generation and verification, respectively; $5.42 \mathrm{~ms}$ for certificate verification. For the time delays, the delay between onboard unit and RSU, the delay between RSU and local authority, the delay between RSU and central authority are 20, 5, and 10 milliseconds, respectively.

Fig. 3(a) shows pseudonym requesting delay comparison between our proposed $P^{3}$ and the traditional pseudonym management scheme proposed in [18]. In F-IoV, vehicles directly request for new pseudonyms from geo-distributed local authorities. Every local authority generates its own pseudonyms and only distributes them to local vehicles for avoiding congestion delay in the network. Localized processing of pseudonym requests can lessen the response time and round-trip delay. Thus, in our scheme, there exists better performance about pseudonym requesting delay for vehicles. Clearly, with more local authorities, the pseudonym requesting delay is reduced because of balanced workloads among local authorities.

We consider a scenario that all the arrival vehicles request for new pseudonyms. With the increasing arrival rate $\lambda$, the advantage of requesting delay is more obvious. For example, the pseudonym requesting delay is $440 \%$ less than that of the existing scheme in [18], when there are 2 local authorities and $\lambda=70$. Meanwhile, along with less pseudonym requesting delay, communication overheads also are reduced due to localized processing for pseudonym requests in Fig. 3(b). With edge deployment of local authorities, these authorities interact with vehicles via "short-fat" communications in our scheme instead of "long-thin" communications in the existing schemes. It is good for the vehicles to avoid larger communication overheads. Besides, the total reduced communication overheads in our scheme increases with the increasing $\lambda$.

Fig. 4 shows the pseudonym entropy comparison of different pseudonym schemes (i.e., our $P^{3}$ and traditional pseudonym management schemes) with respect to different traffic conditions and pseudonym changing cost evaluated by $\lambda$ and $c$, respectively. This figure shows that both denser traffic and lower pseudonym changing cost play a positive effect on improving achieved entropy. For example, when $\lambda=70$ and $c=0.3$, the enhanced pseudonym entropy of our proposed scheme is $8 \%$ higher than that of the existing pseudonym management scheme in [18]. During the period of red light at the intersections, the local authorities can schedule more vehicles to participate in the pseudonym changing game due to the above described less pseudonym requesting delay. Thus, more vehicles choose to change their pseudonyms collectively for improving the pseudonym entropy leading to better privacy protection. Moreover, with the increasing $c$, less vehicles would like to change their pseudonyms in the social hotspots, which brings smaller pseudonym entropy. 


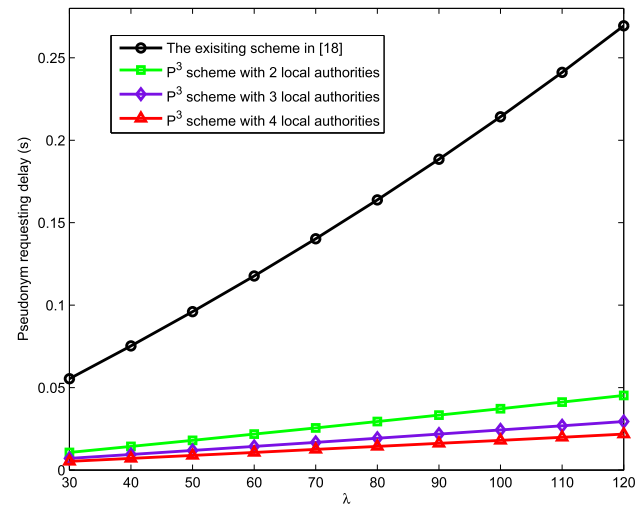

(a)

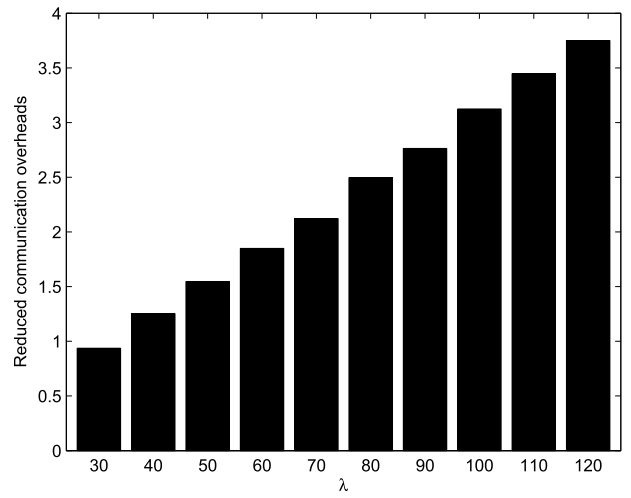

(b)

Fig. 3. Performance comparison about (a) pseudonym requesting delay and (b) reduced communication overheads.

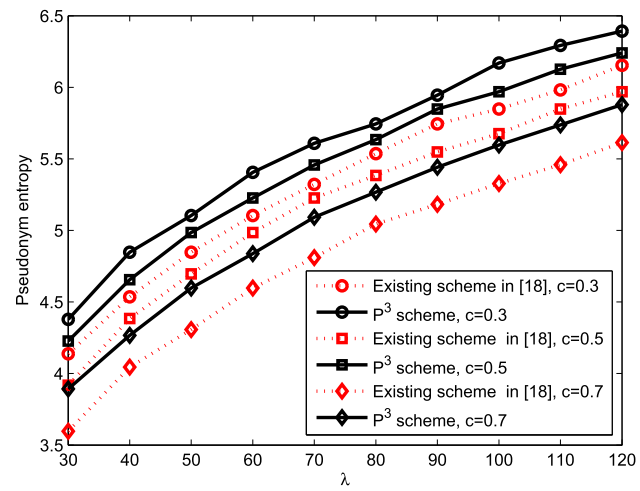

Fig. 4. Achieved entropy comparison of different pseudonym schemes.

Fig. 5(a) and Fig. 5(b) show Nash equilibrium comparison of pseudonym changing game with respect to different pseudonym changing costs and estimation parameters for entropy loss. Here, the mean value of vehicle arrival $\lambda$ is set to be 60 per minute. For a specified social hotspot, we can observe that the game converges fast and the Nash equilibrium is reached after multiple iterations. Besides, when the pseudonym changing cost increases, some vehicles are not willing to change pseudonyms unless the improvement of location privacy at this social hotspot is high enough. So higher pseudonym changing cost results in less vehicles finally choosing to change their pseudonyms at this social hotspot as shown in Fig. 5(a). Fig. 5(b) shows performance impact about the

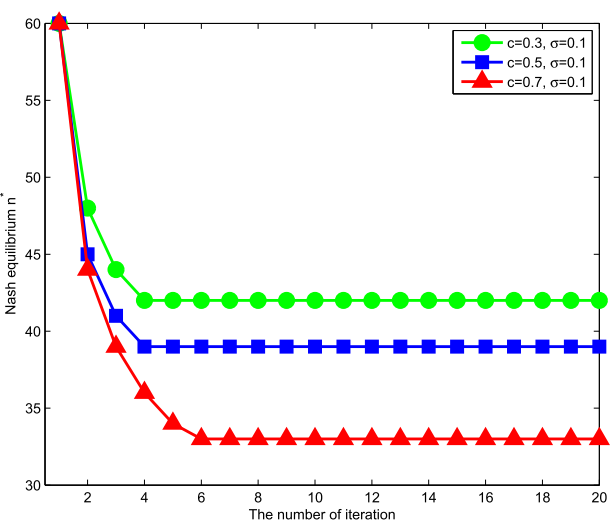

(a)

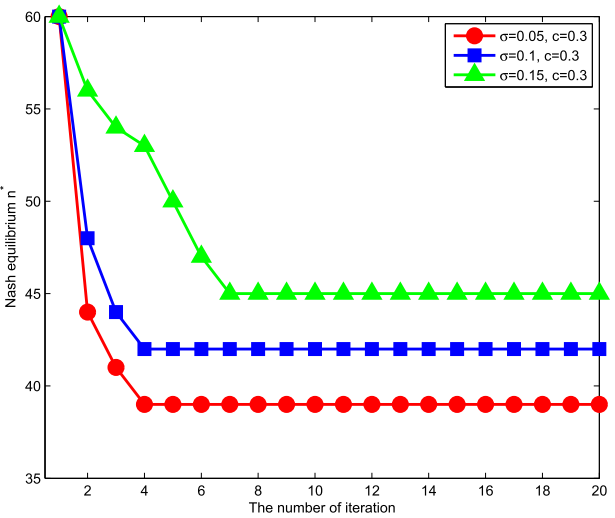

(b)

Fig. 5. Nash equilibrium comparison with respect to different (a) pseudonym changing costs, and (b) standard deviation of entropy loss's estimation.

standard deviation of entropy loss's estimation value (i.e., $\sigma$ ) on Nash equilibrium. The final size of the game $\left(n^{*}\right)$ increases with the decreasing value of $\sigma$. This is because that vehicles have less knowledge of the adversary when $\sigma$ increases. The vehicles are more willing to change the pseudonyms for high enough privacy levels. Thus, the willingness of changing pseudonym $w_{c}$ is encouraged while the willingness of maintaining old pseudonym $w_{m}$ is restrained. From Fig. 5(a) and Fig. 5(b), we note that pseudonym changing cost $c$ and standard deviation of entropy loss's estimation value $\sigma$ have negative impacts on the pseudonym changing game.

In summary, according to the above figures, our proposed $P^{3}$ scheme is efficient for location privacy protection with less communication overhead in F-IoV.

\section{CONCLUSION}

In this paper, we presented a privacy-preserved pseudonym scheme with a hierarchical architecture to achieve pseudonym management at the network edge. Geo-distribution authorities for pseudonym management are deployed in pseudonym fogs. Pseudonyms are generated and distributed to vehicles in time. For secure and efficient pseudonym management, secure communication protocols for privacy-preservation are designed in F-IoV. Finally, we present a context-ware pseudonym changing game for vehicles to change pseudonym with 
context awareness. The security analysis shows that our proposed scheme provides secure communication and privacy preservation for vehicles. Numerical results indicate that the scheme outperforms the existing pseudonym management schemes in terms of improving the vehicles' location privacy and reducing communication overheads.

There is an interesting problem, that the proposed scheme is not very applicable to situations with sparse vehicles, can be further studied. For future work, we will take these situations into consideration, and design enhanced schemes for pseudonym management in F-IoV. We also plan to consider social networks in which the vehicles are involved. Social networks have become pervasive, which leads to a new paradigm in solving pseudonym management problems from social relationships among vehicles.

\section{REFERENCES}

[1] A. Al-Fuqaha, M. Guizani, M. Mohammadi, M. Aledhari, and M. Ayyash, "Internet of Things: A survey on enabling technologies, protocols, and applications," IEEE Commun. Surveys Tuts., vol. 17, no. 4 , pp. 2347-2376, 4th Quart., 2015.

[2] J. Wan et al., "Mobile crowd sensing for traffic prediction in Internet of vehicles," Sensors, vol. 16, no. 1, 2016, Art. no. 88.

[3] Y. Gao et al., "Are cloudlets necessary?" School Comput. Sci., Carnegie Mellon Univ., Pittsburgh, PA, USA, Tech. Rep. CMU-CS-15-139, 2015.

[4] X. Hou, Y. Li, M. Chen, D. Wu, D. Jin, and S. Chen, "Vehicular fog computing: A viewpoint of vehicles as the infrastructures," IEEE Trans. Veh. Technol., vol. 65, no. 6, pp. 3860-3873, Jun. 2016.

[5] R. Yu, J. Kang, X. Huang, S. Xie, Y. Zhang, and S. Gjessing, "MixGroup: Accumulative pseudonym exchanging for location privacy enhancement in vehicular social networks," IEEE Trans. Depend. Sec. Comput., vol. 13, no. 1, pp. 93-105, Jan. 2016.

[6] J. Petit, F. Schaub, M. Feiri, and F. Kargl, "Pseudonym schemes in vehicular networks: A survey," IEEE Commun. Surveys Tuts., vol. 17, no. 1, pp. 228-255, 1st Quart., 2015.

[7] J. Freudiger et al., "On non-cooperative location privacy: A gametheoretic analysis," in Proc. ACM CCS, 2009, pp. 324-337.

[8] J. Freudiger, M. Raya, M. Félegyházi, P. Papadimitratos, and J.-P. Hubaux, "Mix-zones for location privacy in vehicular networks," in Proc. Int. Workshop WiN-ITS, 2007, pp. 1-7.

[9] R. Lu, X. Lin, X. Liang, and X. Shen, "A dynamic privacy-preserving key management scheme for location-based services in VANETs," IEEE Trans. Intell. Transp. Syst., vol. 13, no. 1, pp. 127-139, Mar. 2012.

[10] L. Buttyán, T. Holczer, A. Weimerskirch, and W. Whyte, "SLOW: A practical pseudonym changing scheme for location privacy in VANETs," in Proc. IEEE VNC, Oct. 2009, pp. 1-8.

[11] R. Lu, X. Lin, T. H. Luan, X. Liang, and X. Shen, "Pseudonym changing at social spots: An effective strategy for location privacy in VANETs," IEEE Trans. Veh. Technol., vol. 61, no. 1, pp. 86-96, Jan. 2012.

[12] M. Raya and J.-P. Hubaux, "Securing vehicular ad hoc networks," J. Comput. Secur., vol. 15, no. 1, pp. 39-68, 2007.

[13] S. Jiang, X. Zhu, and L. Wang, "An efficient anonymous batch authentication scheme based on HMAC for VANETs," IEEE Trans. Intell. Transp. Syst., vol. 17, no. 8, pp. 2193-2204, Aug. 2016.

[14] K. Gai, M. Qiu, H. Zhao, L. Tao, and Z. Zong, "Dynamic energy-aware cloudlet-based mobile cloud computing model for green computing," J. Netw. Comput. Appl., vol. 59, pp. 46-54, Jan. 2016.

[15] S. F. Abedin, M. G. R. Alam, N. H. Tran, and C. S. Hong, "A fog based system model for cooperative IoT node pairing using matching theory," in Proc. APNOMS, 2015, pp. 309-314.

[16] S. Biswas et al., "Privacy and anonymity in VANETs: A contemporary study," Ad Hoc Sens. Wireless Netw., vol. 10, nos. 2-3, pp. 177-192, 2010.

[17] N. B. Truong, G. M. Lee, and Y. Ghamri-Doudane, "Software defined networking-based vehicular Adhoc network with fog computing," in Proc. IFIP/IEEE Int. Symp. IM May 2015, pp. 1202-1207.

[18] Y. Park, C. Sur, and K.-H. Rhee, "Pseudonymous authentication for secure V2I services in cloud-based vehicular networks," J. Ambient Intell. Hum. Comput., vol. 7, no. 5, pp. 661-671, 2015.

[19] H. Artail and N. Abbani, "A pseudonym management system to achieve anonymity in vehicular ad hoc networks," IEEE Trans. Depend. Sec. Comput., vol. 13, no. 1, pp. 106-119, Jan. 2016.
[20] R. Suryawanshi et al., "Focusing on mobile users at the edge of Internet of things using fog computing," Int. J. Sci. Eng. Technol. Res., vol. 4 no. 17, pp. 3225-3231, 2015.

[21] C. Song et al., "Limits of predictability in human mobility," Science, vol. 327, no. 5968, pp. 1018-1021, 2010.

[22] Z. Ma, F. Kargl, and M. Weber, "Pseudonym-on-demand: A new pseudonym refill strategy for vehicular communications," in Proc. IEEE Veh. Technol. Conf., Sep. 2008, pp. 1-5.

[23] S. Karumanchi, A. Squicciarini, and D. Lin, "Privacy-aware access control for message exchange in vehicular ad hoc networks," Telecommun. Syst., vol. 58, no. 4, pp. 349-361, 2015.

[24] R. Lu, X. Lin, H. Zhu, P.-H. Ho, and X. Shen, "ECPP: Efficient conditional privacy preservation protocol for secure vehicular communications," in Proc. IEEE INFOCOM, Apr. 2008, pp. 1229-1237.

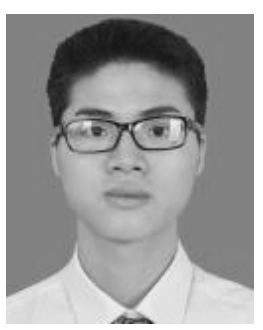

Jiawen Kang received the M.S. degree from the Guangdong University of Technology, China, in 2015, where he is currently pursuing the Ph.D. degree. His research interests mainly focus on resource management, and security and privacy protection in wireless communications and networking.

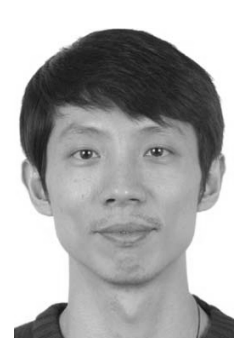

Rong Yu (M'08) received the Ph.D. degree from Tsinghua University, China, in 2007. He was with the School of Electronic and Information Engineering, South China University of Technology. In 2010, he joined the Institute of Intelligent Information Processing, Guangdong University of Technology, where he is currently a Full Professor. He has co-invented over 30 patents and authored or co-authored over 100 international journal and conference papers. His research interest mainly focuses on wireless communications and networking, including cognitive radio, wireless sensor networks, and home networking. He was the member of the Home Networking Standard Committee in China, where he led the standardization work of three standards.

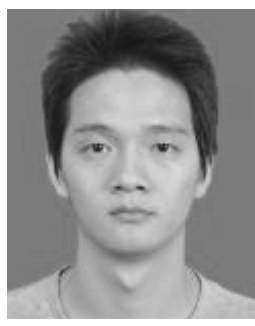

Xumin Huang is currently pursuing the Ph.D. degree in networked control systems with the Guangdong University of Technology, China. His research interests mainly focus on network performance analysis, and simulation and enhancement in wireless communications and networking.

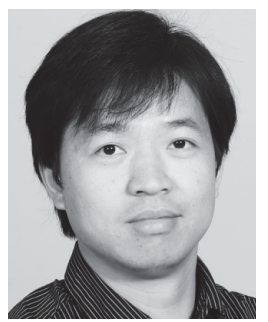

Yan Zhang (SM'10) received the Ph.D. degree from the School of Electrical and Electronics Engineering, Nanyang Technological University, Singapore. He is currently a Full Professor with the Department of Informatics, University of Oslo, Norway. His current research interests include: next-generation wireless networks leading to $5 \mathrm{G}$, green, and secure cyberphysical systems, such as smart grid, healthcare, and transport. He is an IEEE VTS Distinguished Lecturer. He is also a Senior Member of the IEEE ComSoc, the IEEE CS, the IEEE PES, and the IEEE VT society. He is a fellow of the IET. He serves as the Chair for a number of conferences, including the IEEE GLOBECOM 2017, the IEEE VTC-Spring 2017, the IEEE PIMRC 2016, the IEEE CloudCom 2016, the IEEE ICCC 2016, the IEEE CCNC 2016, the IEEE SmartGridComm 2015, and the IEEE CloudCom 2015. He serves as a TPC member for numerous international conferences, including the IEEE INFOCOM, the IEEE ICC, the IEEE GLOBECOM, and the IEEE WCNC. He is an Associate Technical Editor of the IEEE Communications Magazine, an Editor of the IEEE TRANSACTIONS ON GREEN COMMUNICATIONS AND NETWORKING, an Editor of the IEEE COMMUNICATIONS SURVEYS AND TUTORIALS, an Editor of the IEEE INTERnet of Things Journal, and an Associate Editor of IEEE ACCESS. 